\title{
ALGUNOS FACTORES ABIÓTICOS DE DISTRIBUCIÓN DE LOS EFEMERÓPTEROS Y PLECÓPTEROS (INSECTA) EN LAS SIERRAS SEGUNDERA, CABRERA Y TELENO (NW PENÍNSULA IBÉRICA)
}

\author{
M. Vidal López y P. Membiela Iglesia \\ Universidad de Vigo. E. U. de Formación del Profesorado de EGB. Vicente Risco s/n. 32001 ORENSE.
}

Palabras clave: efemerópteros, plecópteros, factores abióticos, distribución, Península Ibérica.

\author{
ABSTRACT \\ SOME ABIOTIC DISTRIBUTION FACTORS OF MAYFLIES AND STONEFLIES (INSECTA) IN SEGUNDERA, \\ CABRERA AND TELENO MOUNTAINS (NW IBERIAN PENINSULA)
}

Some data is given about the distribution of mayflies and stoneflies in the Segundera, Cabrera and Teleno mountains (NW Iberian Peninsula), in relation with some abiotic factors. Between them, altitude and magnitude of the watercourse seem to explain the observed faunistic differences between the sampling localities, whereas sustrate nature and current velocity seem to account for the distribution of some mayfly nymphs in the same sample locality.

\section{INTRODUCCIÓN}

Los principales factores de distribución de los macroinvertebrados bentónicos son, según Hynes (1970), la velocidad de la comente, la temperatura (que comprende los efectos de altitud y período del año) y el sustrato, si ahí se incluye la vegetación y las sustancias disueltas. Otros factores importantes serían la competencia interespecífica, el grado de exposición a la luz y los condicionantes biogeográficos. En una revisión de la biología de los Efemerópteros (Brittain, 1982) se señala la temperatura, el sustrato, la calidad del agua, y en aguas rápidas la velocidad de la corriente como los factores más importantes de distribución. Hynes (1970) en una revisión similar, indicaba que la temperatura, el sustrato, en algunos casos la composición química del agua, las crecidas o la desecación y la alimentación son los factores más importantes en la distribución de las larvas de plecópteros.

Por tanto, se puede considerar como un objetivo importante conocer el poder explicativo de los diversos factores abióticos cn la distribución de los macroinvertebrados bentónicos de las aguas epicontinentales de la Península Ibérica. En esta línea se sitúa el presente estudio, que se inscribe en el marco de los estudios limnológicos sobre el área occidental de la Península Ibérica, zona todavía mal conocida, y es complementario de otros trabajos ya realizados sobre los macroinvertebrados de Galicia (ver MEMBIELA et al., 1990).
Además, se pretende estudiar la influencia de diversos factores abióticos (altitud, magnitud del curso de agua y parámetros asociados, temperatura, $\mathrm{pH}$, oxígeno, conductividad, tipo de sustrato y velocidad de la corriente) en la distribución de los Efemerópteros y Plecópteros en las sierras Segundera, Cabrera y Teleno; un área de la que todavía existen pocos datos (GARCÍA DE JALÓN $\boldsymbol{e} t$ al., 1986; GONZÁLEZ DEL TANAGO y GARCÍA DE JALÓN, 1983 a y b; GONZÁLEZ DEL TANAGO, 1984 a y b; STUDEMAN y TOMKA, 1987; MEMBIELA, 1988).

\section{ÁREA DE ESTUDIO, ESTACIONES DE MUESTREO Y METODOLOGÍA}

El área de estudio comprende las sierras Segundera, Cabrera y Teleno situadas entre la cuenca del Duero y la del Miño-Sil, zona dónde la escasez de población presupone un reducido grado de contaminación, alterado localmente por la explotación de pizarras en la Cabrera y el turismo masivo en la zona del lago de Sanabria (CABERO, 1977).

Se han elegido 11 estaciones (Fig. 1), que se han muestreado con una periodicidad trimestral, mediante la captura de Plecópteros adultos utilizando una red cazamariposas, y larvas de Efemerópteros con la ayuda de un copo tipo Surber de 1/20 $\mathrm{m}^{2}$ de superficie de muestreo y $250 \mu \mathrm{m}$ de luz de malla. En cada estación se recogían tantas muestras de bentos como com- 

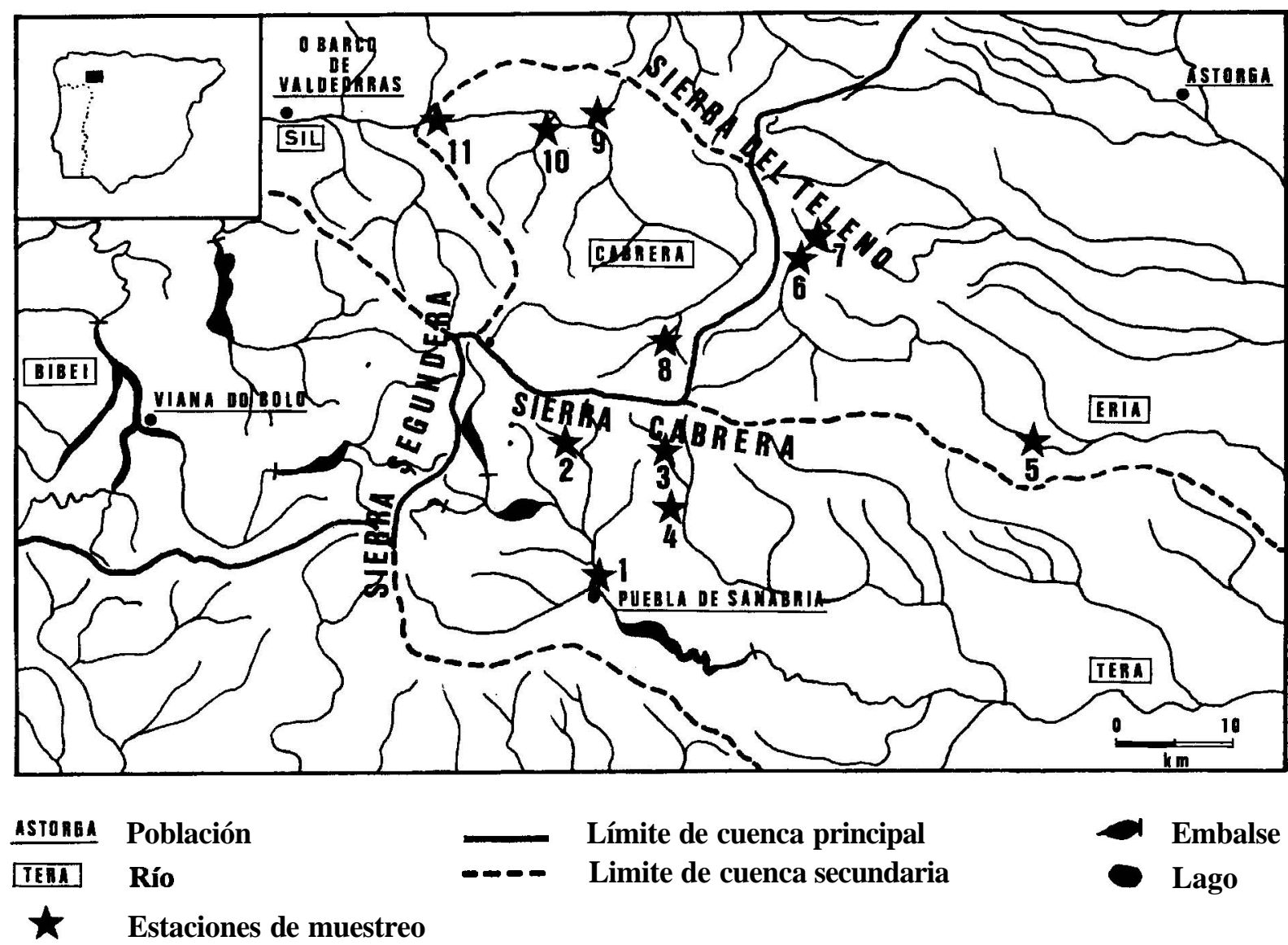

FIGURA. 1.- Area de estudio y estaciones de muestreo.

FIGURE 1.- Study area and sampling localities.

binaciones hubiera, de los distintos tipos de sustrato (macrofitas, musgo, cantos, gravas, arenas y limo) y velocidades de la corriente en superficie, según la escala de BERG (1948) (Muy Lenta $v=0-10$ crnís, Lenta $v=10-25 \mathrm{~cm} / \mathrm{s}$, Moderada $v=25-50$ $\mathrm{cm} / \mathrm{s}$, Rápida $v=50-100 \mathrm{~cm} / \mathrm{s}$, Muy Rápida $v>100 \mathrm{~cm} / \mathrm{s}$ ). Asimismo, se ha realizado el análisis de algunos parámetros fisicoquímicos ( $\mathrm{T}, \mathrm{O}, \mathrm{pH}$, conductividad).

\section{RESULTADOS y DISCUSIÓN}

El presente estudio se basa en un inventario faunístico previamente realizado (VIDAL, 1992), los resultados del análisis de los parámetros físicoquímicos estudiados (Tabla 1), y los escasos datos previamente existentes sobre el área de estudio. No obstante, debido a los problemas de la sistemática larvaria en Plecópteros hemos preferido utilizar solo las citas apoyadas en la captura de adultos.
TABLA 1.- Estaciones de muestreo: coordenadas UTM y valores de diversos parámetros fisicoquímicos. (ES.= estaciones de muestreo, ALT.= altitud, T= temperatura, COND.= conductividad).

TABLE 1.- Sampling localities: UTM coordinates and values of some physicochemical parameters. $(E S .=$ sampling localities, ALT $=$ altitude, $\mathrm{T}=$ temperature, $\mathrm{COND}=$ conductivity).

\begin{tabular}{lcrccrr} 
ES. & $\begin{array}{c}\text { COORD. } \\
\text { UTM }\end{array}$ & $\begin{array}{c}\text { ALT. } \\
(\mathbf{m})\end{array}$ & \multicolumn{1}{c}{ T } & \multicolumn{1}{c}{ pH $\mathbf{C})$} & $\begin{array}{c}\text { OXÍGENO } \\
(\boldsymbol{\%})\end{array}$ & $\begin{array}{c}\text { COND. } \\
(\mu \mathrm{S} / \mathbf{c m})\end{array}$ \\
\hline 1 & 29TPG9659 & 880 & $4.9-18.9$ & $6.8-7.7$ & $101-106$ & $18-28$ \\
2 & 29TPG9271 & 1220 & $4.4-12.7$ & $6.7-7.5$ & $93-109$ & $17-36$ \\
3 & 29TQG0071 & 1160 & $4.3-13.5$ & $6.7-7.6$ & $101-108$ & $9-13$ \\
4 & 29TQG0268 & 1080 & $5.8-14.1$ & $6.8-7.5$ & $100-108$ & $13-16$ \\
5 & 29TQG3174 & 860 & $3.0-17.7$ & $6.9-7.3$ & $95-102$ & $22-25$ \\
6 & 29TQG1289 & 1320 & $5.2-15.7$ & $6.8-7.6$ & $98-119$ & $18-24$ \\
7 & 29TQG1190 & 1400 & $6.4-11.0$ & $6.9-7.4$ & $94-104$ & $35-62$ \\
8 & 29TPG9980 & 1040 & $3.8-14.2$ & $6.7-7.6$ & $98-105$ & $10-18$ \\
9 & 29TPG9497 & 640 & $5.2-15.3$ & $6.5-7.4$ & $101-109$ & $10-30$ \\
10 & 29TPG8999 & 460 & $5.5-15.8$ & $6.4-7.4$ & $98-121$ & $43-83$ \\
11 & 29TPG7998 & 360 & $5.6-19.9$ & $6.3-7.3$ & $104-117$ & $52-98$ \\
\hline
\end{tabular}


Hay que señalar que, con los datos existentes, no se han apreciado variaciones en la distribución de ambos grupos debidas a la temperatura, $\mathrm{pH}$ y conductividad. Sin embargo, el hecho de que la temperatura haya sido medida a diferentes horas del día puede invalidar su aparente falta de relación con la distribución. La concentración de oxígeno de las aguas presenta valores próximos o por encima de la saturación, lo que la elimina como un factor limitante.

El análisis de la distribución de los Efemerópteros y Plecópteros en relación con los factores abióticos estudiados, ha permitido deducir que la altitud y la magnitud del curso de agua y parámetros asociados son los factores que tienen un mayor poder explicativo en las diferencias faunísticas observadas entre distintas estaciones, y que dentro de una misma estación, el tipo de sustrato y la velocidad de la comente en superficie intervienen en la distribución de las larvas de algunas especies de Efemerópteros.

A continuación, pasamos a comentar más detalladamente la distribución de los diferentes taxones de ambos grupos de organismos en relación con los factores abióticos importantes en su distribución (Apéndice 1, Figs. 2 y 3).

\section{Distribución altitudinal}

La gama de altitudes se ha dividido en tres intervalos: zonas bajas (altitud $<500 \mathrm{~m}$ ), zonas medias (altitud entre 500 y 1000 m) y zonas altas (altitud> $1000 \mathrm{~m}$ ).

\begin{tabular}{|c|c|c|c|c|}
\hline EFEMERÓPTEROS & $\begin{array}{l}\text { ALTITUD } \\
\text { (m) }\end{array}$ & $\begin{array}{c}\text { GAMA DE } \\
\text { ALTITUDES }\end{array}$ & $\begin{array}{c}\text { NÚMERO } \\
\text { ORDEN }\end{array}$ & $\begin{array}{l}\text { GAMA CURSOS } \\
\text { DE AGUA }\end{array}$ \\
\hline B. alpinus & $860-1400$ & medias-altas ${ }^{\mathrm{L}}$ & 2 & pequeños \\
\hline B. atrebatinus & $360-950$ & bajas-medias' & 5 & grandes \\
\hline B. estrelensis & $360-1220$ & amplia & $2-5$ & amplia \\
\hline B. fuscatus & $360-1320$ & amplia & $2-5$ & amplia \\
\hline B. melanonyx & $360-1320$ & amplia & $2-5$ & amplia \\
\hline B. muticus & $360-1320$ & amplia & $2-5$ & amplia \\
\hline B. rhodani & $360-1400$ & amplia & $1-5$ & amplia \\
\hline O. rhenana & $360-920$ & bajas-medias' & $4-5$ & grandes \\
\hline D. paradinasi & $460-1320$ & amplia & $2-4$ & medianos \\
\hline E. ignita & $360-1500$ & amplia & $2-5$ & amplia \\
\hline E. iberica & $860-920$ & medias $^{\mathrm{L}}$ & 4 & grandes \\
\hline S. albai & $360-1008$ & bajas-medias' & $4-5$ & grandes \\
\hline C. beskidensis & $460-1040$ & bajas-medias' & $2-4$ & medianos \\
\hline C. luctuosa & $360-1008$ & bajas-medias' & $4-5$ & grandes \\
\hline C. humilis & $640-1400$ & medias-altas ${ }^{\mathrm{L}}$ & $1-4$ & amplia \\
\hline H. eldae & $860-1320$ & medias-altas & $1-4$ & amplia \\
\hline E. lineata & $360-1320$ & amplia & $2-5$ & amplia \\
\hline
\end{tabular}

Del análisis de todos los datos existentes (ver Apéndice 1) se puede deducir la existencia de 6 categorías en la distribución altitudinal: taxones de zonas bajas (1), de zonas bajas-medias (7), de zonas medias (5), de zonas medias-altas (5), de zonas altas (15) y de amplia distribución altitudinal (10). Asimismo se pueden apuntar algunas conclusiones:

- Las dos categorías más numerosas son, la de amplia distribución altitudinal, con una representación mayoritaria de Efemerópteros y la de zonas altas, con una dominancia clara de Plecópteros. El hecho de que los Plecópteros, en conjunto, sean más abundantes en zonas más elevadas que los Efemerópteros, ya ha sido señalado previamente por diversos autores (KAMLER, 1965; BERTHELEMY, 1966; MACAN, 1974).

- Existe un elevado número de taxones (29) que presentan, en el área de estudio, una distribución altitudinal diferente a la encontrada en zonas limítrofes, taies como Galicia (PUIG, 1983; PARDO et al., 1991; MEMBIELA, en prensa), cuenca del Duero (GONZÁLEZ DEL TANAGO, 1984a), norte de Portugal (CORTÉS et al., 1986; CORTÉS, 1989) o en otras zonas peninsulares (ver Apéndice 1).

\begin{tabular}{|c|c|c|c|c|}
\hline PLECÓPTEROS & $\begin{array}{l}\text { ALTITUD } \\
\text { (m) }\end{array}$ & $\begin{array}{c}\text { GAMA DE } \\
\text { ALTITUDES }\end{array}$ & $\begin{array}{c}\text { NÚMERO } \\
\text { ORDEN }\end{array}$ & $\begin{array}{r}\text { CURSOS } \\
\text { DE AGUA }\end{array}$ \\
\hline B. arcuata & 1080 & $\operatorname{altas}^{1}$ & $1^{2}$ & pequeños \\
\hline T. schoenemundi & 360 & bajas' & 5 & grandes \\
\hline P. hispanica & $1040-1320$ & altas $^{L}$ & $2-3$ & medianos \\
\hline P. i. iberiaca & 1220 & altas' & $2^{2}$ & pequeños \\
\hline P. navacerrada & $1080-1400$ & altas & $1-2$ & pequeños \\
\hline P. p. asturica & $980-1400$ & altas $^{1}$ & $1-2^{2}$ & pequeños \\
\hline A. guadarramensis & $980-1400$ & $\operatorname{altas}^{1}$ & 2 & pequeños \\
\hline L. alosi & $1220-1320$ & altas $^{\mathrm{L}}$ & $2^{2}$ & pequeños \\
\hline L. castillana & $1040-1320$ & altas' & $2-3^{2}$ & medianos \\
\hline L. franzi & $1040-1110$ & altas $^{1}$ & $2-3^{2}$ & medianos \\
\hline L.f.fusca & $360-860$ & bajas-medias $^{1}$ & $4-5^{\prime}$ & grandes \\
\hline L. geniculata & $360-880$ & bajas-medias & $4-5^{2}$ & grandes \\
\hline L. hiberiaca & 1320 & altas & 2 & pequeños \\
\hline L. hispanica & $1040-1320$ & altas $^{1}$ & 2 & pequeños \\
\hline L. lusitanica & $640-1220$ & medias-altas' & $2-3^{\prime}$ & medianos \\
\hline L. madritensis & $980-1320$ & altas' & $2-3^{2}$ & medianos \\
\hline L. maroccana & $360-1400$ & amplia & $1-5$ & amplia \\
\hline L stupeningi & $980-1400$ & altas' & $1-2^{2}$ & pequeños \\
\hline C. libera & $360-1400$ & amplia & $1-5$ & amplia \\
\hline C. mitis & $860-1040$ & medias $^{1}$ & $2-4$ & medianos \\
\hline I. grammatica & 860 & medias $^{\mathrm{L}}$ & $4^{2}$ & grandes \\
\hline H. flaviventris & 860 & medias' & $4^{2}$ & grandes \\
\hline P. marginata & $640-860$ & medias' & $3-4^{2}$ & medianos \\
\hline Ch. acuta & $1040-1220$ & altas $^{1}$ & 2 & pequeños \\
\hline Ch. tripunctata & 1220 & altas' & $2^{\prime}$ & pequeños \\
\hline S. torrentium & $880-1320$ & medias-altas $^{1}$ & $2-4^{2}$ & medianos \\
\hline
\end{tabular}

APÉNDICE 1.- Distribución en relación con la altitud y magnitud del curso de agua de los Efemerópteros y Plecópterosen el área de estudio.

${ }^{1}$ Distribución altitudinal diferente en otras zonas de la Península Ibérica.

Preferencia por otro curso de agua en otras zonas de la Península Ibérica. 
- La altitud parece ser uno de los factores abióticos decisivos en la distribución de ambos grupos de insectos. Este factor ya ha sido señalado como clave en su distribución por muchos otros autores (HYNES, 1941; AUBERT, 1946; MACAN, 1974; HYNES, 1976; BRITTAIN, 1982; WARD, 1986). El efecto altitud es consecuencia de la influencia de factores primarios en la distibución, fundamentalmente temperatura y alimentación (KNIGHT y GAUFIN, 1966; WARD, 1982), pues al variar la altitud se produce un cambio en el régimen térmico de las aguas y una variación en la cantidad de materia autóctona y alóctona presentes. También pueden sufrir cambios la presión de predación y las interacciones competitivas (WARD, 1982).

\section{Distribución en relación con la magnitud del curso de agua}

Aunque la utilización del número de orden en expresiones relativas a tamaños de cursos de agua ha sido criticada (HUGHES y OMERMIK, 1983), hemos seguido el criterio de MONZON et al. (1991) de utilizar el número de orden como la variable morfométrica más explicativa en la comparación del tamaño de diferentes cursos de agua.

El criterio seguido ha sido considerar cursos de agua pequeños (número de orden 1-2), medianos (número de orden 2-3-4) y grandes (número orden 4-5).

Del análisis de los datos (ver Apéndice 1), se puede deducir la existencia de 4 categorías según la preferencia de Efemerópteros y Plecópteros por la magnitud del curso de agua: taxones de pequeños cursos de agua (12), de entidad media (10), de gran entidad (10) y taxones con amplia distribución (11). Asimismo, se pueden apuntar algunas conclusiones:

- Existe una clara preferencia por parte de algunos taxones por un determinado curso de agua, hecho ya señalado por diversos autores (ULFSTRAND, 1968; LILLEHAMMER, 1974).

- La categoría taxones de cursos de agua pequeños esta constituida mayoritariamente por Plecópteros, y la categoría de amplia distribución por Efemerópteros.

- Existe un número considerable de taxones (16) con diferencias en su preferencia por el curso de agua en relación con otras zonas peninsulares.

\section{Influencia del tipo de sustrato y velocidad de la corriente sobre la distribuciónde las larvas de Efemerópteros}

El análisis de los datos existentes sobre la influencia del tipo de sustrato (Fig. 2) ha permitido deducir dos tipos de compor- tamiento. Así, mientras algunas especies han sido encontradas en único tipo, caso de O. rhenana y $\mathrm{C}$. beskidensis en CANTOS+GRAVAS+ARENAS y D. paradinasi en MUSGO+CANTOS; otras especies, tales como B. rhodani y E. igni$t a$, aparecen en sustratos diversos.

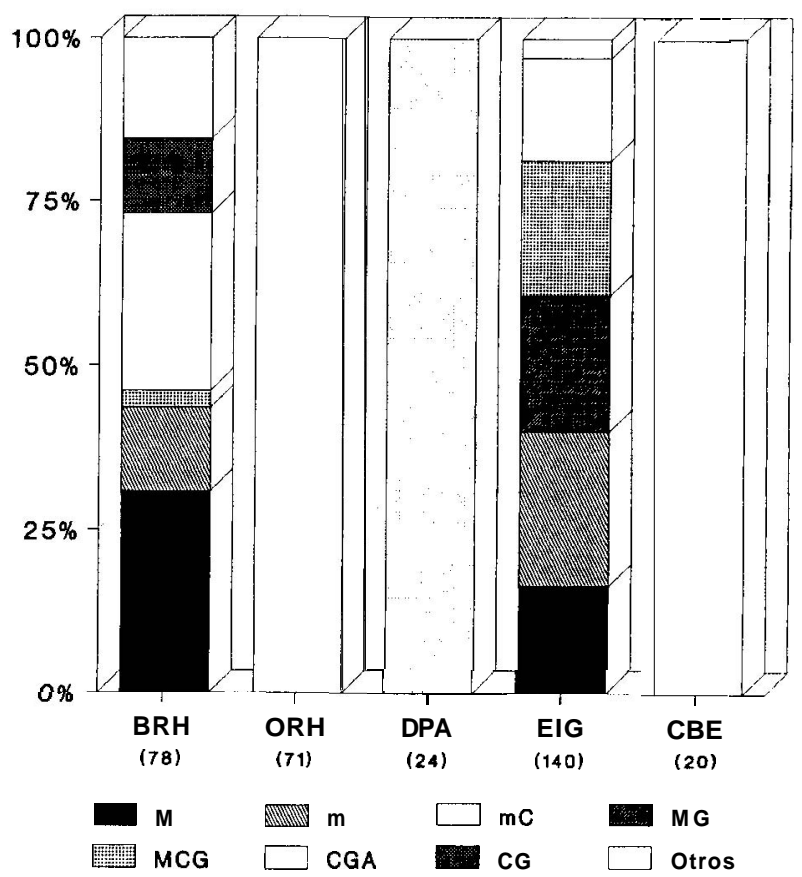

FIGURA 2.- Preferendum de las larvas de diversas especies de Efemerópteros por el sustrato $(M=$ macrofitas; $m=$ musgo; $C=$ cantos; $G=$ gravas; $A=$ arenas). Entre paréntesis se señala el número de individuos. $\mathrm{BRH}=\mathrm{B}$. rhodani, $\mathrm{ORH}=\mathrm{O}$. rhenana, DPA= D. paradinasi, $\mathrm{EIG}=\mathrm{E}$. ignita y $\mathrm{CBE}=\mathrm{C}$. beskidensis. FIGURE. 2.- Preference by sustrate of some mayfly nymphs (M= macrophytes; $\mathrm{m}=$ moss; $\mathrm{C}=$ stones; $\mathbf{G}=$ gravels; $\mathrm{A}=$ sand). Number of captures in brackets. $\mathrm{BRH}=\mathrm{B}$. rhodani, $\mathrm{ORH}=\mathrm{O}$. rhenana, $\mathrm{DPA}=\mathrm{D}$. paradinasi, $\mathrm{EIG}=\mathrm{E}$. ignita $\mathrm{y}$ $\mathrm{CBE}=\mathrm{C}$. beskidensis

En relación con la velocidad de la corriente (Fig. 3), se debe indicar que algunas muestran preferencia por una gama determinada, caso de C. luctuosa por velocidad LENTA-MUY LENTA, C. beskidensis por MODERADA, B. fuscatus, B. rhodani, O. rhenana y D. paradinasi por RÁPIDA-MUY RÁPIDA. Por contra, E. ignita no muestra preferencia clara por ningún tipo de velocidad determinado.

Del análisis anterior se pueden deducir algunas conclusiones y comentarios:

- La naturaleza del sustrato y la velocidad de la comente son dos factores importantes en la distribución dentro de una misma estación. Este hecho ha sido ya previamente señalado en numerosos estudios (HYNES, 1970; MACAN, 1974; CORKUM et al., 1977; BRITTAIN, 1982; MINSHALL, 1984). 


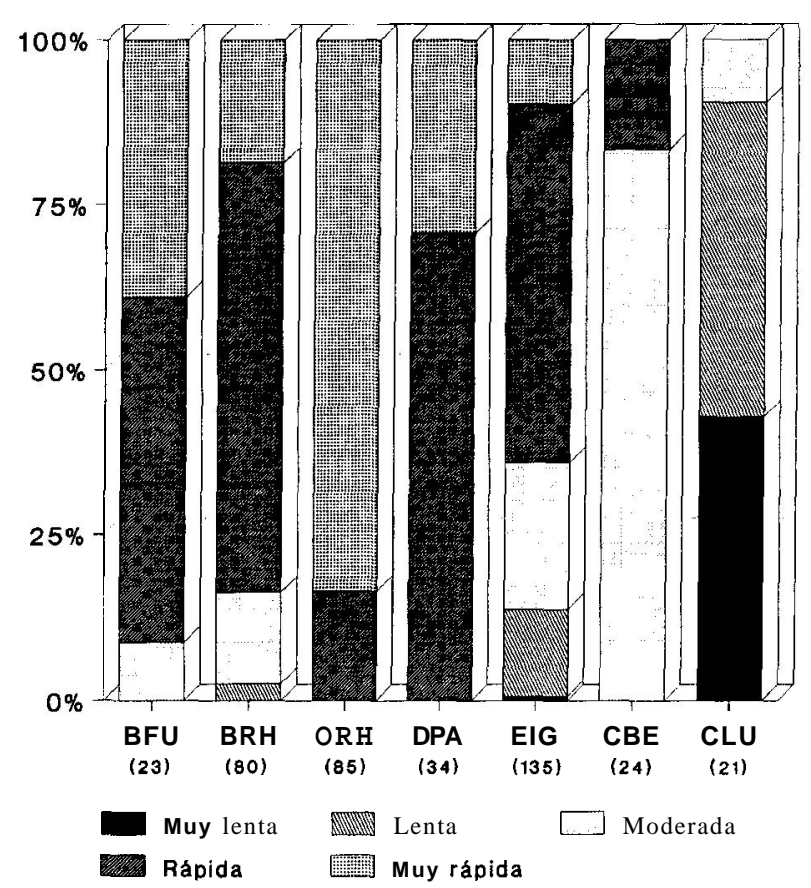

FIGURA 3.- Preferendum de las larvas de diversas especies de Efemerópteros por la velocidad de la comente en superficie según la escala de BERG (1948). Entre paréntesis se señala el número de individuos. $\mathrm{BFU}=\mathrm{B}$. fuscatus, $\mathrm{BRH}=\mathrm{B}$. rhodani, $\mathrm{ORH}=\mathrm{O}$. rhenana, $\mathrm{DPA}=\mathrm{D}$. paradinasi, $\mathrm{EIG}=\mathrm{E}$. ignita, $\mathrm{CBE}=\mathrm{C}$. beskidensis y $\mathrm{CLU}=\mathrm{C}$. luctuosa.

FIGURA. 3.- Preference by surface current velocity of some mayfly nymphs according to BERG (1948) scale. Number of captures in brackets. BFU $=$ B. fuscatus, $\mathrm{BRH}=\mathrm{B}$. rhodani, $\mathrm{ORH}=0$. rhenana, $\mathrm{DPA}=\mathrm{D}$. paradinasi, $\mathrm{EIG}=\mathrm{E}$. ignita, $\mathrm{CBE}=\mathrm{C}$. beskidensis y $\mathrm{CLU}=\mathrm{C}$. luctuosa.

- En la mayoría de los casos, los taxones han sido capturados en el mismo tipo de sustrato o velocidad de la corriente que en otras zonas peninsulares.

- D. paradinasi presenta en el área de estudio un preferendum por el tipo de sustrato distinto al señalado por STUDEMAN y TOMKA (1987) en diversas zonas de la Península Ibérica, dónde ha sido encontrada siempre asociada a Oenanthe crocata $\mathrm{L}$.

\section{Comentario global}

Se ha hecho un análisis de las diferencias y similitudes entre estaciones por un lado, y por otro, entre los diferentes taxones de Efemerópteros y Plecópteros capturados, aplicando el paquete estadístico NTSYS-pc versión 1.0 a la matriz de datos de presencia-ausencia estaciones-especies.

Se han calculado los coeficientes de similaridad entre estaciones y entre especies, utilizando el coeficiente de JACCARD (1902), y a la correspondiente matriz se le ha aplicado un pro- cedimiento de agrupación (cluster)SAHN (secuencial aglomerativo jerárquico y sin solapamiento) mediante el método UPGMA (método medias aritméticas con pares no contrapesados).

De un primer análisis realizado a partir de la fauna de Efemerópteros y Plecópteros en las 11 estaciones muestreadas (Fig. 4), se puede deducir la existenciade tres grupos de estaciones:

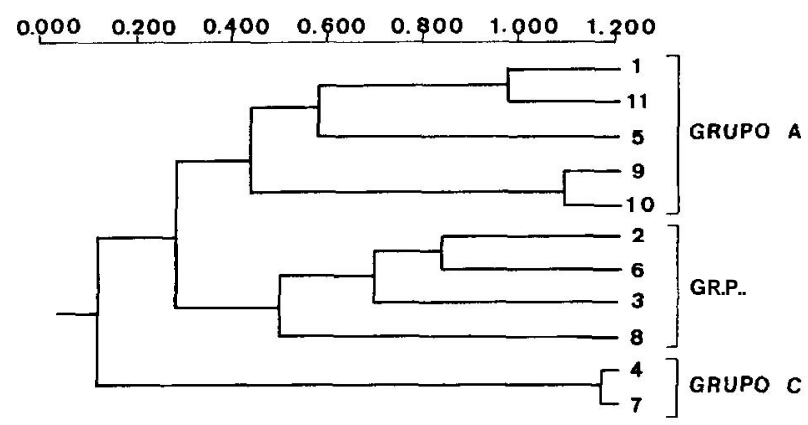

FIGURA. 4.- Agrupación de las estaciones realizada a partir de la matriz de similaridad estaciones-taxones (Efemerópteros y Plecópteros).

FIGURE. 4.- Cluster of the localities realize since the similarity matrix localitiestaxa (Ephemeroptera and Plecoptera).

- GRUPO A.- Cursos de agua de tamaño medio-grande situados en zonas de altitud baja-media: estaciones $1,5,9,10$ y 11 .

- GRUPO B.- Cursos de agua de tamaño medio localizados en zonas altas: estaciones 2, 3, 6 y 8 .

- GRUPO C.- Cursos de agua de tamaño pequeño situados en zonas altas: estaciones 4 y 7.

Aplicando el mismo método se ha efectuado un segundo análisis para observar el agrupamiento de los distintos taxones capturados. Así, a partir de la matriz de similaridad entre taxones, considerando conjuntamente la fauna de Efemerópteros y Plecópteros, obtenemos un total de 8 grupos (Fig. 5). No obstante, la elevada semejanza existente entre varios de estos grupos entre sí, en cuanto a su distribución en relación con la altitud y magnitud del curso de agua, aconseja limitar su número a solo cuatro grupos:

- GRUPO I (EP1, EP2, EP5, EP6).- Taxones de zonas medias y/o altas y cursos de agua de tamaño pequeño y/o medio (18): B. alpinus, B. arcuata, P. hispanica, P. i. iberiaca, $P$. navacerrada, $P$. pyrenaica asturica, A. guadarramensis, $L$. alosi, L. castillana, L. franzi, L. hiberiaca, L. hispanica, L. lusitanica, L madritensis, $L$ stupeningi, Ch. acuta, Ch. tripunctata y S. torrentium.

- GRUPO II (EP3, EP4).- Taxones de distribución relativamente amplia en relación con la altitud y/o el tamaño del curso de agua (13): B. estrelensis, B. fuscatus, B. melanonyx, B. muticus, B. rhodani, D. paradinasi, E. ignita, C. beskidensis, $C$. humilis, H. eldae, E. lineata, L. maroccana y C. libera. 
$\begin{array}{lllllll}0.000 & 0.200 & 0.400 & 0.600 & 0.800 & 1.000 & 1.200\end{array}$

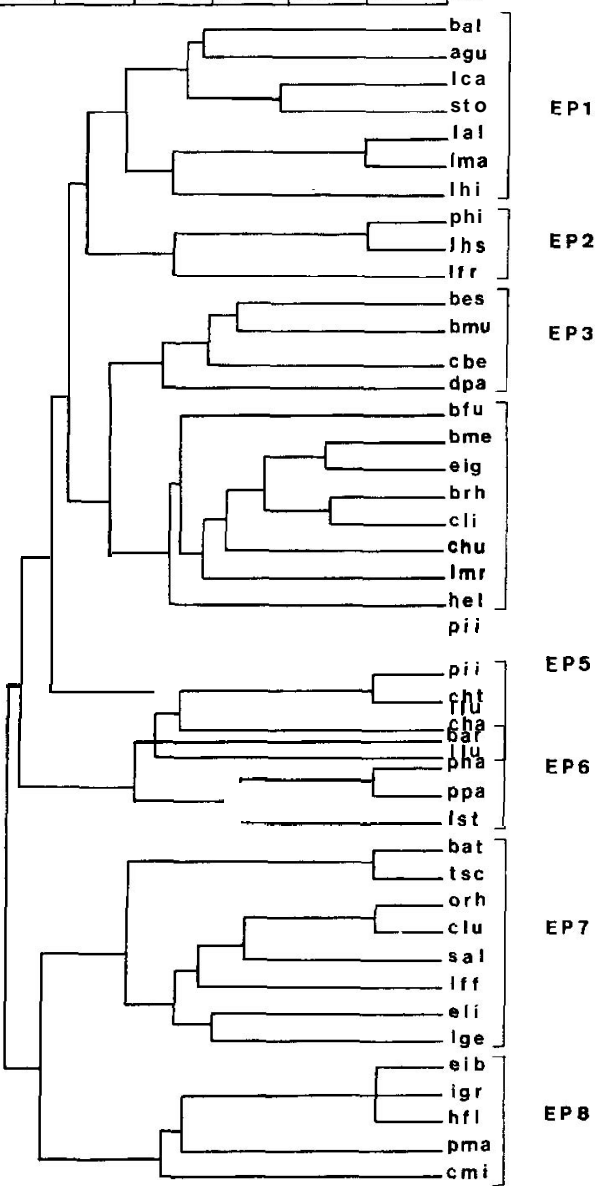

FIGURA. 5.- Agrupación de los taxones realizado a partir de la matriz de similaridad estaciones-taxones (Efemerópteros y Plecópteros). AGU= A. guadarramensis, $\mathrm{BAL}=\mathrm{B}$. alpinus, $\mathrm{BAR}=\mathrm{B}$. arcuata, $\mathrm{BAT}=\mathrm{B}$. atrebatinus, $\mathrm{BES}=\mathrm{B}$. estrelensis, $\mathrm{BFU}=\mathrm{B}$. fuscatus, $\mathrm{BME}=\mathrm{B}$. melanonyx, $\mathrm{BMU}=\mathrm{B}$. muticus, $\mathrm{BRH}=\mathrm{B}$. rhodani, $\mathrm{CBE}=\mathrm{C}$. beskidensis, $\mathrm{CHU}=\mathrm{C}$. humilis, $\mathrm{CLU}=\mathrm{C}$. luctuosa, $\mathrm{CHA}=\mathrm{Ch}$ acuta, $\mathrm{CHT}=\mathrm{Ch}$. hipunctata, $\mathrm{CLI}=\mathrm{C}$. libera, $\mathrm{CMI}=\mathrm{C}$. mitis, $\mathrm{DPA}=\mathrm{D}$. paradinasi, $\mathrm{EIB}=\mathrm{E}$. iberica, $\mathrm{EIG}=\mathrm{E}$. ignita, $\mathrm{ELI}=\mathrm{E}$. lineata, $\mathrm{HEL}=\mathrm{H}$. eldae, $\mathrm{HFL}=\mathrm{H}$ flaviventris, $\mathrm{IGR}=\mathrm{I}$. grammatica, $\mathrm{LAL}=\mathrm{L}$. alosi, $\mathrm{LCA}=\mathrm{L}$. castillana, $\mathrm{LFR}=\mathrm{L}$. franzi, $L F F=L$. fusca fusca, $L G E=L$. geniculata, $L H I=L$. hiberiaca, $L H I=L$. his panica, $\mathrm{LLU}=\mathrm{L}$. lusitanica, $\mathrm{LMA}=\mathrm{L}$. madritensis, $\mathrm{LMA}=\mathrm{L}$. maroccana, $\mathrm{LST}=$ L. stupeningi, $\mathrm{ORH}=\mathrm{O}$. rhenana, $\mathrm{PHI}=\mathrm{P}$. hispanica, $\mathrm{PII}=\mathrm{P}$. i iberiaca, $\mathrm{PMA}=\mathrm{P}$. marginata, $\mathrm{PNA}=\mathrm{P}$. navacerrada, $\mathrm{PPA}=\mathrm{P}$. p. asturica, $\mathrm{SAL}=\mathrm{S}$. albai, $\mathrm{STO}=\mathrm{S}$ torrentium y TSC $=\mathrm{T}$. schoenemundi.

FIGURE. 5.- Cluster of the taxa realize since the similarity matrix localities-taxa (Ephememptera and Plecoptera). $\mathrm{AGU}=\mathrm{A}$. guadarramensis, $\mathrm{BAL}=\mathrm{B}$. alpinus, $\mathrm{BAR}=\mathrm{B}$. arcuata, $\mathrm{BAT}=\mathrm{B}$. atrebatinus, $\mathrm{BES}=\mathrm{B}$. estrelensis, $\mathrm{BFU}=\mathrm{B}$. fuscatus, $\mathrm{BME}=\mathrm{B}$. melanonyx, $\mathrm{BMU}=\mathrm{B}$. muticus, $\mathrm{BRH}=\mathrm{B}$. rhodani, $\mathrm{CBE}=\mathrm{C}$. beskidensis, $\mathrm{CHU}=\mathrm{C}$. humilis, $\mathrm{CLU}=\mathrm{C}$. luctuosa, $\mathrm{CHA}=\mathrm{Ch}$. acuta, $\mathrm{CHT}=\mathrm{Ch}$. tripunctata, $\mathrm{CLI}=\mathrm{C}$. libera, $\mathrm{CMI}=\mathrm{C}$. mitis, $\mathrm{DPA}=\mathrm{D}$. paradinasi, $\mathrm{EIB}=\mathrm{E}$. iberica, $\mathrm{EIG}=\mathrm{E}$. ignita, $\mathbf{E L I}=\mathrm{E}$. lineata, $\mathrm{HEL}=\mathrm{H}$. eldae, $\mathrm{HFL}=\mathrm{H}$. flaviventris, $\mathrm{IGR}=\mathrm{I}$. grammatica, $L A L=L$. alosi, $L C A=L$. castillana, $L F R=L$. franzi, $L F F=L$. fusca fusca, $\mathrm{LGE}=\mathrm{L}$. geniculata, $\mathrm{LHI}=\mathrm{L}$. hiberiaca, $\mathrm{LHI}=\mathrm{L}$. hispanica, $\mathrm{LLU}=\mathrm{L}$. lusitanica LMA $=\mathrm{L}$. madritensis, $\mathrm{LMA}=\mathrm{L}$. maroccana, $\mathrm{LST}=\mathrm{L}$. stupeningi, $\mathrm{ORH}=\mathbf{O}$. rhenana, $\mathrm{PHI}=\mathrm{P}$. hispanica, $\mathrm{PII}=\mathrm{P}$. i iberiaca, $\mathrm{PMA}=\mathrm{P}$. marginata, $\mathrm{PNA}=\mathrm{P}$. navacerrada, $\mathrm{PPA}=\mathrm{P}$. p. asturica, $\mathrm{SAL}=\mathrm{S}$. albai, $\mathrm{STO}=\mathrm{S}$. torrentium y $\mathrm{TSC}=\mathrm{T}$. schoenemundi.
- GRUPO III (EP7).- Taxones de zonas bajas y/o medias en cursos de agua relativamente grandes (7): B. atrebatinus, O. rhenana, $S$. albai, $C$. luctuosa, T. schoenemundi, $L$ fusca fusca y $L$ geniculata.

- GRUPO IV (EP8).- Taxones de zonas medias y/o altas ausentes de los cursos de agua de tamaño relativamente muy grande (Orden 5) o muy pequeño (Orden 1) (5): E. iberica, C. mitis, I. grammatica, $H$. flaviventris y $P$. marginata.

Aunque tras el procedimiento de clasificación E. lineata aparece en el grupo EP7 (Ver Fig. 5), sus datos de distribución (Apéndice 1) aconsejan su inclusión en el Grupo IV. Además, se debe señalar que no se ha muestreado ningún curso de agua de tamaño pequeño en zonas bajas o medias, ni evidentemente tampoco de tamaño grande en zonas altas.

Este análisis nos permite confirmar el poder explicativo de la altitud y el tamaño del curso de agua en la distribución de ambos grupos de organismos en el área de estudio. Si comparamos la distribución de los Efemerópteros y Plecópteros en el área de estudio (Fig. 6) podemos deducir algunas conclusiones:

NUMERO DE TAXONES

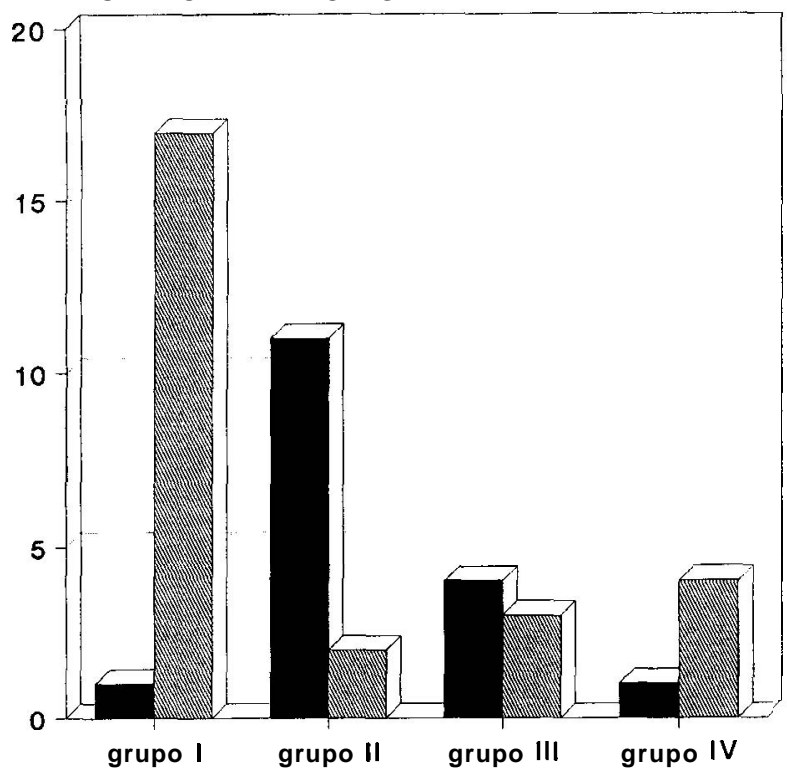

FIGURA. 6.- Asociaciones de Efemerópteros y Plecópteros en relación con la altitud y la magnitud del curso de agua. Gmpo I= taxones de zonas medias y/o altas y cursos de agua de tamaño pequeño y/o medio; grupo 11= taxones de distribución relativamenteamplia en relación con la altitud y/o el tamaño del curso de agua; grupo III= taxones de zonas bajas y/o medias en grandes cursos de agua; gmpo $I V=$ taxones situados en zonas medias $y / o$ altas ausentes de los cursos de agua de tamaño muy grande o muy pequeño.

FIGURE. 6.- Asociations of mayflies and stoneflies in relation with watercourse magnitude and altitude. Group I= taxons of middle and/or high zones and small and/or middle watercourses; group 11= taxons with relatively wide distribution in altitude and/or watercourse size; group III= taxons of low and/or middle zones in large watercourses, group IV= taxons in middle and/or high zones absent from large or small watercourses. 
- Los Efemerópteros son un grupo constituido fundamentalmente por especies de amplia distribución en relación con la magnitud del curso de agua y la altitud (Grupo II), o bien por especies presentes en cursos de agua relativamente grandes situados en zonas bajas y/o medias (Grupo III).

- La mayoría de las especies de Plecópteros, aparecen en cursos de agua de tamaño pequeño y/o medio situados en zonas medias y/o altas (Grupo I).

\section{BIBLIOGRAFÍA}

AUBERT, J., 1946. Les Plécoptères de la Suisse Romande. Mitt. Schweiz. Ent. Ges., 20: 8-128.

BERG, K., 1948. Biological studies on the River Sussa. Folia Limnol. Scand., 4: 1-318.

BERTHELEMY, C., 1966. Recherches écologiques et biogéographiques sur les Plécoptères et Coléoptères d'eau courante (Hydraena et Elminthidae) des Pyrénées. Annls Limnol., 2(2): 227-458.

BRITTAIN, J.E., 1982. Biology of mayflies. Ann. Rev. Entomol., 27: 119-147.

CORKUM, L.D.; POINTING, P.J. \& J.J.H. CIBOROWSKI, 1977. The influence of current velocity and substrate on the distribution and drift of two species of mayflies (Epherneroptera). Can. J. Zool., 55: 1970-1977.

CABERO, V., 1977. Morfología glaciar y deterioro ecológico en la Sierra Segundera: el lago de Sanabria. V Coloquio de Geografía (Granada): 257-269.

CORTÉS, R.M., 1989. Biotipologia de ecossistemas lóticos do nordeste de Portugal. Tesis Doctoral. Universidade de Trasos-Montes e Alto Douro. Vila Real.

CORTÉS, R.M.; TJARDA, K. \& A. SIMOES, 1986. Estudio de un río de montaña de una zona granítica del norte de Portugal. Limnética 2: 197-204.

GARCÍA DE JALÓN, D.; GONZÁLEZ DEL TANAGO, M.; BARCELÓ, E.; MONTES, C.; MENES, F. \& C. CASADO, 1986. Contribución al estudio faunístico de algunas taxocenosis de insectos acuáticos (Epherneroptera, Plecoptera, Trichoptera, Coleoptera y Heteroptera) del Parque Natural del Lago de Sanabria y Río Tera (Zamora). Actas de las VIII Jornadas A. e E., Sevilla : 1145-1156.

GONZÁLEZ DEL TANAGO, M., 1984a. Distribución y biología de la familia Baetrdae (Ephem.) en la Cuenca del Duero. Bol. Asoc. esp. Entom., 8: 73-94.

GONZÁLEZ DEL TANAGO, M., 1984b. Distribution of Plecopterain the Duero Basin (Spain). Annls Limnol., 20 (112): 49-55. GONZÁLEZ DEL TANAGO, M. \& D. GARCÍA DE JALÓN, 1983a. New Ephemerellidae from Spain (Epherneroptera). Aquatic Insects 5(3): 147-156.
GONZÁLEZ DEL TANAGO, M. \& D. GARCÍA DE JALÓN, 1983b. The Oligoneuriidae (Ephemeroptera) of the Duero Basin (Central North Spain). Arch. Hydrobiol., 97(3): 395-405.

HUGHES, R.M. \& J.M. OMERNIK, 1983. An alternative for characterizing stream size. In: Dynamic of lotic ecosystems (Fontaine, T.D. \& S.M. Bartell eds.): 87-101. Ann Arbor Science Publishers. Michigan.

HYNES, H.B.N., 1941. The taxonomy and ecology of the nymphs of the British Plecoptera with notes on the adults and eggs. Trans. Roy. Ent. Soc., London, 91: 459-557.

HYNES, H.B.N., 1970. The Ecology of Running Waters. Liverpool University Press. Liverpool.

HYNES, H.B.N., 1976. Biology of Plecoptera. Ann. Rev. Entom., Palo Alto, 21: 135-153.

JACCARD, P., 1902. Lois de distribution florale dans la zone alpine. Bull. Soc. Vaud. Sc. Nat., 38: 69-130.

KAMLER, E., 1965. Thermal conditions in mountain waters and their influence on the distribution of Plecoptera and Ephemeroptera larvae. Ekol. pol. A, 13: 377-414.

KNIGHT, A.W. \& A.R. GAUFIN, 1966. Altitudinal distribution of stoneflies (Plecoptera) in a Rocky Mountain drainage system. J. Kans. Entomol. Soc., 39: 668-675.

LILLEHAMMER, 1974. Norwegian stoneflies. II. Distribution and relationship to the environment. Norsk. ent. Tidsskr., 21: 195-250.

MACAN, T.T., 1974. Freshwater ecology. 2" ed, Longman. Londres.

MEMBIELA, P., 1988. Los Plecópteros de Galicia: estudio faunístico y ecológico. Tesis Doctoral. Universidad de Santiago de Compostela. Santiago de Compostela.

MEMBIELA, P., en prensa. Inventario dos Plecópteros de Galicia. Cadernos da Area de Ciencias Biolóxicas. Publicacions do Seminario de Estudos Galegos.

MEMBIELA, P.; COBO, F.; GONZALEZ, M. \& E. MARTINEZ-ANSEMIL, 1990. A investigación limnolóxica en Galicia con especial referencia ós macroinvertebrados: precedentes, estado actual e perspectivas. Ingenium 2: 81-94.

MINSHALL, G.W., 1984. Aquatic insect-substratum relationships. In: The Ecology of Aquatic Insects (Resh, V.H. y D.M Rosenberg eds.): 358-400. Praeger Publishers. New York.

MONZÓN, A.; CASADO, C.; MONTES, C. \& D. GARCÍA DE JALÓN, 1991. Organizaciónfuncional de las comunidades de rnacroinvertebrados acuáticos de un sistema fluvial de montaña (SistemaCentral, río Manzanares, España). Limnética 7: 97-112.

PARDO, I.; EIROA, E. \& F. NOVOA, 1991. New records of Mayflies from Galicia (Epherneroptera). In: Overview and Strategies of Ephemeroptera and Plecoptera (Alba Tercedor, J. 
y A. Sanchez Ortega eds.): 289-296. The Sandhill Crane Press, Inc. Florida.

PUIG, M.A., 1983. Contribución al conocimiento de los Efemerópteros del río Tambre (La Coruña). Actas del I Congreso Ibérico de Entomología, León, II: 603-607.

STUDEMAN, D. \& I. TOMKA, 1987. Contribution to the study of European Ephemerellidae (Ephemeroptera). I. Completion of description of three endemic Iberian species. Mitt. Schweiz. Entomol. Ges., 60(3/4): 361-378.
ULFSTRAND, S., 1968. Benthic animal communities in Lapland streams. Oikos, Suppl., 10: 1-120.

VIDAL, M., 1992. Efemerópteros y Plecópteros de las sieras Segundera, Cabrera y Teleno. Tesis de Licenciatura. Universidad de Vigo. Ourense.

WARD, J.V. 1982. Altitudinal zonation of Plecoptera in a Rocky Mountain stream. Aquatic Insects 4(2): 105-110.

WARD, J.V., 1986. Altitudinal zonation in a Rocky Mountain stream. Arch. Hydrobiol./Suppl., 74: 133-199. 